\title{
An Investigation on the Patients Undergoing Kidney Stone Open Surgery: A Single-Center Report From Sistan and Baluchistan Province, Iran
}

\author{
Amin Afshari Moghaddam ${ }^{1 *}$, Salehe Badini ${ }^{2}$ \\ ${ }^{1}$ Faculty of Medicine, Zabol University of Medical Sciences, Zabol, Iran \\ ${ }^{2}$ Student Research Committee, Zabol University of Medical Sciences, Zabol, Iran
}

*Correspondence to

Amin Afshari Moghaddam, Faculty of Medicine, Zabol University of Medical Sciences, Zabol, Iran,

Email: amn_afsharim@yahoo.com

Received June 5, 2021

Accepted June 25, 2021

Published online June 30, 2021

Please cite this article as follows: Afshari Moghaddam A. An investigation on the patients undergoing kidney stone open surgery: a single-center report from Sistan and Baluchistan Province, Iran. Int J Basic Sci Med. 2021;6(2):6468. doi:10.34172/ ijbms.2021.11.

\begin{abstract}
Introduction: Urinary stones are among major urological diseases. Open kidney surgery has always been of historical importance in the management of most urinary stones, especially in complicated cases. The aim of this study was to investigate the cases of open kidney stone surgery in Amir Al-Momenin hospital of Zabol in 2016-2017.

Method: In the present descriptive cross-sectional study, the patients who underwent open kidney stone surgery in Amir Al-Momenin hospital were evaluated. The investigated variables included gender, age, changes in blood pressure, postoperative complications, and duration of surgery. The information was collected from hospital records and entered into SPSS software for analysis.

Results: In this study, 25 patients were studied, of whom $14(56 \%)$ were females. The mean age of the patients was $37.84 \pm 17.15$ years. The youngest and oldest patients were 1 and 66 years old, respectively. Twelve patients (48\%) had staghorn stones, and the rest (13 patients) had other types of stones. Blood pressure remained unchanged in $80 \%$ of the patients while $12 \%$ developed hypertension. Postoperative fever and infection were observed in only $4 \%$ of the patients. The prevalence of staghorn stones in men and women were $45.5 \%$ and $50 \%$, respectively. The difference was not statistically significant $(P=0.821)$.

Conclusion: The present study showed that the most common stones leading to open kidney surgery were of the staghorn type. Males were more likely to undergo open kidney surgery than females.

Keywords: Renal stones, Open surgery, Surgical complications
\end{abstract}

\section{Introduction}

Nephrolithiasis or kidney stone disease is a common, painful and costly disease. The resulting medical costs in the United States are estimated to be more than US $\$ 2$ billion per year. According to the research conducted in Iran and based on clinical findings, 87 people per 100000 population in Iran are annually diagnosed with stone-forming kidneys, with the highest prevalence of stones in the northwestern and western regions of the country. ${ }^{1}$

Urinary tract stones are the third most common urinary tract disease after urinary tract infection and prostate abnormalities. ${ }^{2}$ Surgery was the main method for treating kidney stone before the invention of lithotripsy. However, today only $1 \%$ to $2 \%$ of patients undergo surgery usually when the stone does not respond to other treatments or to correct anatomical abnormalities in the kidney and improve urinary drainage. Surgical procedures include nephrolithotomy (cutting the kidney and removing the stone), nephrectomy (if kidney function is lost due to infections or hydronephrosis), pyelolithotomy (for renal pelvis stones), urolithotomy or transurethral lithotripsy (stone crushing via the duct and ureteral), and cystotomy (for bladder stones). For bladder stones, it is possible to send an instrument to the bladder through the urethra and crush the stone (cystolitholapaxy). ${ }^{3}$

Open surgery to remove kidney stones has been documented in ancient Greek and

(C) 2021 The Author(s); Published by Zabol University of Medical Sciences. This is an open-access article distributed under the terms of the Creative Commons Attribution License (http://creativecommons.org/licenses/by/4.0), which permits unrestricted use, distribution, and reproduction in any medium, provided the original work is properly cited. 
Roman civilizations, and many of these cases date back to the 1500s and 1600s. Since then, surgical procedures for urinary stones have undergone significant changes by the incorporation of technological innovations and advancements. Prior to these advances and over the past two decades, open surgery was the only treatment for removing kidney stones. ${ }^{4}$

The introduction of percutaneous nephrolithotomy (PNL), extracorporeal shock wave lithotripsy, and urethroscopy have had promising achievements for treating urinary tract stones over the recent years. The indications for open or laparoscopic stone surgery are considerably limited due to advances in PNL, and often open surgery is the second or third treatment option. There is a consensus that the most complex stones, including partial and complete staghorn stones, should be treated primarily with PNL. However, open and laparoscopic surgery may be appropriate if percutaneous and endourological methods are not successful. Indications for surgical intervention to remove kidney stones are: stone growth, high-risk stone-developing patients, kidney obstruction, infections, symptomatic stones, stone size $>15 \mathrm{~mm}$, stones below $15 \mathrm{~mm}$ in the patients who do not adhere to treatment, the patient's preference and social status, and the failure of other treatment modalities. ${ }^{5}$

In open surgery to remove a kidney stone, the surgeon makes an incision on the patient's side to access the kidney and remove the stone. A small tube (drain) is then placed near the kidney, creating a pathway for the discharge of secretions until the kidney heals. The patient will be hospitalized for 3 to 6 days and can usually resume normal activities after 4 to 6 weeks. The complications of open kidney stone surgery include severe bleeding, urinary tract infection, wound infection, the risks associated with anesthesia, and the risk of hernia at the incision site. The success of open surgery depends on the location of the kidney stone; however, this method usually removes the stone completely. ${ }^{6}$ According to a study in Europe and North America, the surgical removal of kidney stones is only indicated in cases with complicated stones or after the failure of lithotripsy, skin nephrolithotomy, or urethroscopy. However, in developing countries where endourological methods are not readily available, these techniques are still important.

Overall, treatment options for kidney and urinary tract stones have evolved significantly over the past few decades, so that almost any stone can be treated in a non-invasive or minimally invasive manner including shock wave lithotripsy, uroscopy, and cutaneous nephrolithotomy. The safety of these techniques compared to traditional methods is desirable; however, they require unique skills, access to instrumentation with relatively high maintenance costs, which potentially limit their use on a global scale. With the advent of endourology, significant advances have been made in laparoscopic surgical techniques so that almost any open surgery can be performed in a minimally invasive laparoscopic procedure.

Urinary stones are among the most common diseases of the urinary system and constitute a high percentage of urology referrals. Therefore, studying the epidemiological characteristics of patients with urinary tract stones and examining the most appropriate treatment measures in these patients are essential. A high percentage of patients who can undergo PNL surgery and be treated with fewer complications are treated with open surgery methods. ${ }^{8}$ Our aim in this study was to investigate the patients undergoing open surgery for kidney stone removal in a hospital in South-east of Iran.

\section{Patients and Methods}

This was a cross-sectional-descriptive study performed on the patients undergoing open kidney stone surgery in Amir Al-Momenin hospital of Zabol, Sistan and Baluchistan province of Iran, in 2016-2017.

\section{Sample Size Calculation and Sampling Method}

All the patients who underwent open kidney stone surgery in Amir Al-Momenin hospital within the study period were included in the study following the accessible sampling method.

\section{Data Collection Tools and Study Protocol}

Data were collected using a researcher-made checklist to document patients' information from their clinical records. After coordination with the hospital's managers, the researcher referred to the HIS department and collected the file of the patients who underwent open surgery to remove kidney stones in 2016-2017. The necessary information was collected, and if more information was needed, the patients, or their physicians, were contacted using the registered phone numbers.

\section{Statistical Analysis}

SPSS version 22 software was used to analyze the data. Data were described with the mean and frequency indices and displayed using tables. The independent student $t$ test was used to compare the means of variables between different groups, and the Spearman correlation coefficient was used to examine the relationship between quantitative variables.

\section{Results}

In this study, 25 patients were studied, of whom 14 (56\%) were males. The mean age of the patients was $37.8 \pm 17.15$ years. The youngest and oldest patients were 1 and 66 years old, respectively. Overall, 12 patients $(48 \%)$ had staghorn stones and the rest (13 patients) had other types of stones. Ten patients (40\%) underwent surgery on the right side and the rest (15 patients) on the left side. Table 1 shows the demographic and clinical information of the 
patients.

Regarding post-operative changes, blood pressure remained unchanged in $20(80 \%)$ of patients, $3(12 \%)$ developed hypertension, and 2 (8\%) developed hypotension in postoperative recovery. Vomiting and wound infection were not observed in any of the patients while urinary tract infection was observed in one of the patients (4\%), who was a 1 -year-old boy with a $5 \mathrm{~mm}$ non-staghorn stone in the left side, who also developed postoperative fever. Table 2 shows the patients' clinical findings after surgery.

Overall, staghorn stones were recovered from 12 patients while others had other types of stones. According to Table 3, the frequency of staghorn stones was not associated with gender $(P=0.821)$, mean surgery duration $(P=0.852)$, and occasions of receiving pain-killers $(P=0.067)$, hospitalization duration $(P=0.538)$. There was no statistically significant correlation between stone size and the duration of surgery $(r=0.169, P=0.420)$.

Table 1. The Patients' Demographic and Clinical Information

\begin{tabular}{lcccc}
\hline Variables & Mean & SD & Min & Max \\
\hline Age $(\mathrm{y})$ & 37.8 & 17.15 & 1 & 66 \\
Hospitalization period $(\mathrm{d})$ & 5.7 & 2.3 & 3 & 16 \\
Anesthesia time $(\mathrm{h})$ & 3.3 & 1 & 2 & 6 \\
Surgery time $(\mathrm{h})$ & 2.7 & 0.99 & 1.3 & 5.3 \\
Stone size $(\mathrm{mm})$ & 39.9 & 16.4 & 5 & 60 \\
Number of received packed cells & 0 & 0 & 0 & 0 \\
Occasions of receiving pain killers & 14.8 & 4.9 & 3 & 22 \\
\hline
\end{tabular}

Table 2. Post-operative Clinical Information in the Studied Patients

\begin{tabular}{lccc}
\hline Variables & & Frequency & Percent \\
\hline \multirow{2}{*}{ Blood pressure } & Unchanged & 20 & 80 \\
& Hypertension & 3 & 12 \\
& Hypotension & 2 & 8 \\
Urinary tract infection & Present & 1 & 4 \\
& Absent & 24 & 96 \\
Post-operative fever & Present & 1 & 4 \\
& Absent & 24 & 96 \\
\hline
\end{tabular}

Also, no significant correlation was observed between stone size and the mean occasion of receiving pain killers ( $\mathrm{r}=0.017, P=0.937)$.

\section{Discussion}

In this study, we found that the majority of patients who underwent surgery for urinary tract stones were men. In a 2017 study on the patients referred for open surgery for kidney stones, Çakici et al stated that the ratio of men to women was 13 to $5 .{ }^{9}$ Also, in a study by Paik et al, males were more common among the patients undergoing open kidney surgery. ${ }^{10}$ These studies are consistent with the present study. However, in a study by Ozgor et al, this ratio did not show a significant deviation despite the fact that men were still more likely to be candidates for surgery. ${ }^{11}$ The reason for the higher prevalence of urinary stones in males may be due to the higher secretion of calcium oxalate and uric acid and less citrate production in men than women, leading to more severe complications. ${ }^{12}$

In the present study, almost half of the patients were identified with staghorn stones. In the study of Çakici et $\mathrm{al}^{9}$ and Aydemir et $\mathrm{al},{ }^{13}$ the most common stones leading to open surgery were staghorn stones. Other studies by Ozgor et $\mathrm{al}^{11}$ and El-Husseiny \& Buchholz ${ }^{14}$ also showed that the most common stones leading to open surgery were staghorn types. Actually, the renal structural changes created by staghorn stones in most cases force the physician to consider surgery to remove the stone. ${ }^{14}$

In the present study, postoperative complications such as wound infection and vomiting were not observed in patients, and only one person developed urinary tract infection and fever, which could be explained by his young age (1 year). In the study of Çakici et al, they reported a low prevalence of complications following surgery, ${ }^{9}$ which was consistent with our study. However, in another study by Yan and Wei, in addition to urinary tract infection and fever, wound infection was also observed in a small number of patients. ${ }^{15}$ Among the most important factors determining the risk of post-operative complications are the surgeon's skill, the procedure's efficiency, and the type of sterilization of surgical instruments. ${ }^{16}$ Overall, it can be said that urological surgeries are safe in terms of complications.

Table 3. The Distribution of Staghorn Kidney Stones Based on Different Demographic and Clinical Variables

\begin{tabular}{|c|c|c|c|}
\hline \multirow{3}{*}{ Variables } & \multicolumn{2}{|c|}{ Kidney Stone Type } & \multirow{3}{*}{$P$ Value } \\
\hline & Staghorn $(n=12)$ & Other $(n=13)$ & \\
\hline & No. $(\%)$ & No. $(\%)$ & \\
\hline \multirow{2}{*}{ Gender } & $7(50)$ & $7(50)$ & \multirow{2}{*}{0.821} \\
\hline & $5(45.5)$ & $6(54.5)$ & \\
\hline Mean surgery duration $(h$, mean $\pm \mathrm{SD}$ ) & $2.6 \pm 0.8$ & $2.8 \pm 1.1$ & 0.852 \\
\hline Occasions of receiving pain killers (mean \pm SD) & $16.67 \pm 4.36$ & $13.08 \pm 4.8$ & 0.067 \\
\hline Hospitalization duration ( $h$, mean \pm SD) & $5.25 \pm 0.865$ & $6.23 \pm 3.166$ & 0.538 \\
\hline
\end{tabular}


Although the majority of our patients did not experience changes in blood pressure, $20 \%$ of them developed either hypertension or hypotension after surgery, which merits further investigations. In the present study, we noticed that older people were more prone to changes in blood pressure, suggesting that these people should be more closely examined for hemodynamic stability and underlying cardiovascular diseases before undergoing stone surgery. In one study, Rowley and Wolf stated that age was an important risk factor for open kidney surgery. ${ }^{17}$ In fact, advanced age has always been a relative risk factor for surgery due to the instability of the cardiovascular condition during surgery ${ }^{18}$ Therefore, it is recommended to accurately monitor blood pressure during open kidney stone surgery, especially in the elderly.

In our study, gender was not significantly associated with the type of stone. Nevertheless, Costa et al. in their study reported that gender was related to the type of stone. ${ }^{18}$ On the other hand, the type of stone had no effects on the durations of surgery and hospitalization in our study. In another study, it was stated that complex stones could prolong the surgery. ${ }^{19}$ We also found that the type of stone did not affect patients' needs for additional analgesia. The size of the stone also had no effect on the duration of the surgery or the need for taking pain killers. As previously noted, one of the main factors influencing the amount of analgesia consumption and the duration of surgery is the surgeon's skill in reducing the rate of tissue damages and complications. ${ }^{20}$

\section{Limitations}

The limitations of this study include the impossibility of performing PNL in our hospital, incomplete files, the lack of follow-up of the patient after discharge, and the low sample size. In addition, the number of the patients who experienced different complications was small, so it was not amenable to effectively investigate postoperative outcomes and late complications (stone recurrence, etc.). One reason for the low prevalence of postoperative complications in the present study was the selection of low to moderate risk patients for surgery.

\section{Conclusion}

Our study showed that the prevalence of staghorn stones in Sistan region was relatively high. However, neither the type nor the size of stones was associated with clinical outcomes such as the length of hospitalization, duration of surgery, and the need for analgesics administration. It is recommended to perform studies with larger sample sizes in the future. Elderly patients should be monitored in terms of cardiovascular condition before performing stone surgery.

\section{Ethical Approval}

In this study, all procedures were in accordance with the national ethical guidelines in research on human subjects.

Competing Interests

There is no conflict of interest to declare.

Acknowledgment

Thanks to Ali Bazi for editing and revising the manuscript.

\section{References}

1. Rafiei H, Malekpoor F, Amiri M, Rahimi Madiseh M, Lalegani H. Kidney stone development among older adults in Iran. J Indian Acad Geriatr. 2014;10:10-13.

2. Ghasemi Basir HR, Mosavi Bahar SH. A survey of epidemiologic factors in patients with urinary stones in Hamadan. Avicenna J Clin Med. 2016;23(2):157-163. [Persian].

3. Parmar MS. Kidney stones. BMJ. 2004;328(7453):14201424. doi:10.1136/bmj.328.7453.1420

4. Paik ML, Resnick MI. Is there a role for open stone surgery? Urol Clin North Am. 2000;27(2):323-331. doi:10.1016/ s0094-0143(05)70261-5

5. Moe OW. Kidney stones: pathophysiology and medical management. Lancet. 2006;367(9507):333-344. doi:10.1016/s0140-6736(06)68071-9

6. Agrawal MS, Singh SK, Singh H. Management of multiple/ staghorn kidney stones: open surgery versus PCNL (with or without ESWL). Indian J Urol. 2009;25(2):284-285.

7. BorofskyMS, LingemanJE. Therole of open andlaparoscopic stone surgery in the modern era of endourology. Nat Rev Urol. 2015;12(7):392-400. doi:10.1038/nrurol.2015.141

8. Tavakkoli Tabassi K, Mohammadi S, Madani A. Investigation patients with urinary stones from the aspect of epidemiologic parameters admitted at urology department of Imam Reza hospital during years 20052008 [Persian]. Alborz Univ Med J. 2013;2(4):197-204. doi:10.18869/acadpub.aums.2.4.197

9. Çakici ÖU, Ener K, Keske M, et al. Open stone surgery: a still-in-use approach for complex stone burden. Cent European J Urol. 2017;70(2):179-184. doi:10.5173/ ceju.2017.1205

10. Paik ML, Wainstein MA, Spirnak JP, Hampel N, Resnick MI Current indications for open stone surgery in the treatment of renal and ureteral calculi. J Urol. 1998;159(2):374-379. doi:10.1016/s0022-5347(01)63922-3

11. Ozgor F, Kucuktopcu O, Ucpinar B, et al. The effects of previous open renal stone surgery types on PNL outcomes. Can Urol Assoc J. 2016;10(7-8):E246-E250. doi:10.5489/ cuaj. 3687

12. Lieske JC, Rule AD, Krambeck AE, et al. Stone composition as a function of age and sex. Clin J Am Soc Nephrol. 2014;9(12):2141-2146. doi:10.2215/cjn.05660614

13. Aydemir H, Budak S, Kumsar Ş, Köse O, Sağlam HS, Adsan Ö. Efficacy of surgical techniques and factors affecting residual stone rate in the treatment of kidney stones. Turk J Urol. 2014;40(3):144-149. doi:10.5152/tud.2014.40360

14. El-Husseiny T, Buchholz N. The role of open stone surgery. Arab J Urol. 2012;10(3):284-288. doi:10.1016/j. aju.2012.03.004

15. Yan SB, Wei Q. [Open stone surgery: is it justified in the era of minimally invasive therapies?]. Zhonghua Wai Ke Za 
Zhi. 2009;47(4):244-247.

16. Chung KC, Kotsis SV. Complications in surgery: root cause analysis and preventive measures. Plast Reconstr Surg. 2012;129(6):1421-1427. doi:10.1097/ PRS.0b013e31824ecda0

17. Rowley MW, Wolf JS Jr. Risk factors for conversion to hand assisted laparoscopy or open surgery during laparoscopic renal surgery. J Urol. 2011;185(3):940-944. doi:10.1016/j. juro.2010.10.063

18. Feringa HH, Bax JJ, Karagiannis SE, et al. Elderly patients undergoing major vascular surgery: risk factors and medication associated with risk reduction. Arch Gerontol Geriatr. 2009;48(1):116-120. doi:10.1016/j. archger.2007.11.003

19. Lechevallier E, Traxer O, Saussine C. [Open surgery for upper urinary tract stones]. Prog Urol. 2008;18(12):952954. doi:10.1016/j.purol.2008.09.013

20. Costa-Bauzá A, Ramis M, Montesinos V, et al. Type of renal calculi: variation with age and sex. World J Urol. 2007;25(4):415-421. doi:10.1007/s00345-007-0177-4 